\title{
The Study of Grid Management Application on Bank Zone
}

\author{
Chen Liyan
}

Wenzhou Business College

\begin{abstract}
Keywords: Grid, Grid Management, Bank.
\end{abstract}
\begin{abstract}
At present, the electronic level of Bank is continuously improving. Grid management, a new managerial concept, has the potential of being a powerful weapon that affects the study on Bank Zone. This paper starts from the connotation and characteristics of grid management, and discusses its structure and content. Then points out the support structure of grid five-layer hourglass system, basic process and implementation content of grid management. Based on these, this paper discusses grid management application in banking industry, including financial grid construction of resource sharing infrastructure, emergency human resource allocation and employee behavioral grid management. This paper furtherly improves the theoretical basis of grid management and provides theoretical support and reference Suggestions for the grid management application in banking industry, which is of great practical significance for the operation and development of Banks.
\end{abstract}

\section{银行网格化管理及其应用探析}

陈丽燕

温州商学院, 温州, 浙江, 中国

关键词：网格; 网格化管理; 银行

中文摘要. 网格很有可能成为未来计算机技术的主要发展方向, 由此网格化管理很有可能将 成为未来新型社会的组织和管理模式, 当前电子化程度不断提高的银行实施网格化管理也是 应此大趋势。本文从网格及网格化管理的内涵和特征出发, 探讨网格化管理的结构和内容, 重点指出了网格五层沙漏体系的结构与支撑、网格化管理的基本流程及实施内容。基于此探 讨网格化管理在银行业的应用, 主要选取资源共享基础设施的金融网格体系构建、应急人力 资源调配、员工行为网格化管理等三大方面。本文进一步完善了网格化管理的理论基础, 并 为银行业的网格化管理应用提供理论支撑和借鉴建议, 对银行的经营和发展具有重要的现实 意义。

\section{1. 引言}

关于网格, 学术界较为认同的有以下几点。一是新的概念, 其目标是实现资源共享和协 同事务; 二是新的技术，是解决多个层系中资源共享和协同事务的新技术和新标准，并把互 联网从通信和信息交互的平台提升成资源共享的平台; 三是高级计算基础设施, 是当前不可 或缺的IT基础设施。网格的应用已在生物制药、高性能计算、城市管理、电子政务管理等方 面取得了重大的成就。而随着网格应用到企业日益推广, 网格化管理这一新模式也日渐被管 理者接受。银行业作为当今信息社会基础性服务行业也不例外，紧跟时代步伐实施网格化管 理, 对内可实施系统资源共享金融网格和员工行为网格, 对外可为信息社会提供优质化和多 样化的金融服务。

\section{2. 网格与网格化管理概述}




\section{1 网格}

网格（Grid）是构筑于互联网上的一组新兴技术，其主要目标是实现网络虚拟环境下的 高性能资源共享和协同事务, 消除信息孤岛和资源孤岛。网格的重要作用则在于以合理的方 式 “粘合” 分散在网络上的信息及信息存储、处理能力，以形成多样化的立体信息网络。

\section{2 网格化管理}

基于新兴信息技术的网格, 网格化管理则是一种新的管理模式, 实践创新与理论创新均 尚为不足。关于网格化管理的定义, 目前学术界持有相对一致的观点。一般认为, 网格化管 理是指借用计算机网络管理思想, 按照一定标准将管理对象划分成若干网格单元, 利用现代 信息技术和各网格单元间的协调机制, 促成各个网格单元之间的有效信息交流, 透明地共享 组织资源，最终以期整合组织资源，并提升管理效率的现代化管理思想。

与传统条块管理思想不同，网格化管理属于集合和开放式管理思想。网格化管理实现统 一服务、资源共享以及协同解决, 一方面讲求更高监督力度, 另一方面也由于高度集中性方 便了监管。网格化管理通过建立独立的监督部门, 标准化网格节点权责并监督运营过程, 以 提高管理效率。统一监管在保障网格化管理的健康运营发挥着重要作用, 通过考察实施状况 和目标状态间的差距和问题, 实现网格化管理的监督、改进、再监督、再改进的螺旋式发展。

网格化管理规范、清晰、高效、创新、集成。在实际运作过程中，员工网格化管理在发 现、处理、考核与监督、法规与体制等层面均可能存在诸多交叉甚至冲突，应以员工满意度 为出发点, 以风控为核心, 充分整合各类管理资源和力量, 以最大限度发挥网格化管理效益。

\section{3. 网格化管理的结构与内容}

\section{1 五层沙漏体系的结构与支撑}

网格化管理并非是平面的一层网格单元和节点, 而是分为五层沙漏结构, 从底部到顶部 分别为构造层、连接层、资源层、汇聚层和应用层, 并且任何下层的组件都可以用为构建上 层组件的基础。这五层并没有严格的划分标准, 更无法完成所有必要的协议排列, 只能定义 出这种结构中每一部分组件的共同要求, 并把这些组件形成一个层次结构的关系。此外, 越 底层越接近具体的物理资源，越顶层越接近抽象的共享资源。

最底层的构造层具有可以支持更先进的共享操作的特定物理资源或者逻辑资源, 其基本 功能包括发现资源的结构状态、控制服务质量和资源管理能力等; 连接层则为资源通信能力 的安全设置重要通信和协议, 能使构造层的资源间交换和流通数据, 实现连接层相互联系和 操作的基本功能; 资源层位于连接层的通信和协议之上, 其资源仅为单个且相对独立, 实现 的是单个资源的共享, 但并未考虑到全局状态和分布式资源集合; 汇聚层聚集单个资源, 以 资源形式协调解决多个资源之间的问题, 强调资源的共性, 并不具体设计具体资源的具体特 点; 位于最上层的应用层, 存在于一个虚拟的组织中, 其功能是寻找下层相应的资源来供给 虚拟组织以期解决虚拟组织中出现的问题。下面四层任意层次提供所需的服务, 应用层则构 造出相应的虚拟组织, 解决相应的具体问题。

\section{2 网格化管理基本流程}

网格化管理的基本流程大致可抽象为五个方面。首先是业务受理, 受理同一平台上网格 内所有用户需求申请, 并审核需求申请和初步分类, 其中网格化管理中心可处理的需求申请 直接转发处理, 涉及到节点组织的需求申请则转到业务分派中心; 二是业务分派, 涉及到节 点组织的业务, 统一根据其类型进一步识别和细分, 并分派到节点上的相应组织进行处理; 三是业务处理, 节点组织受理业务, 并根据具体需求完成业务; 四是服务提交, 体现为网格 化管理的信息披露, 任务执行组织将任务完成信息或进度信息直接反馈给用户, 或至受理中 
心处由受理中心向用户提供反馈; 五是监督检查, 监管部门和用户对收到的服务结果进行确 认和评价, 实现对业务流程和节点组织的监督管理, 且应包括对事后监督部门的监督管理。

\section{3 网格化管理实施内容}

网格化管理的具体实施应以特定领域为单位，根据该领域的行业规范和业务流程，结合 网格化管理的特征和流程来制定具体实施内容，同时又必须不失一般性。网格化管理在各领 域共通的实施内容主要包括六点。

首先是网络化信息传输渠道，网格化管理的高效依赖于网格各节点信息流的畅通，非网 络化的信息传输不利于多组织的信息同步传递; 二是构建资源信息数据库, 网格化管理的社 会资源整合与共享需要强大的数据库及搜索引擎, 因此可建立网格资源分类数据库, 以数字 形式记录网格节点及资源信息; 三是提升用户服务质量, 网格化管理基于用户至上的理念, 应为用户设计更丰富、更快捷的网格化管理系统访问渠道, 并不断优化用户体验; 四是模块 化业务职能, 网格化管理需要对组织职能进行模块化封装, 集中服务属性的公共职能以响应 全局调度, 做到节点职能明确, 促成流程共享; 五是规范化系统和流程协议, 网格化管理系 统的兼容性和开放性依赖于各类协议的规范化建设, 因而需建立合理的业务流程规范、参与 者权责规范以及信息系统规范，有利于资源共享和业务协同的顺利实现; 六是组织业务流程 重组，可包括重组和优化内部业务流程、信息流、服务流及资金流等主要流程。

\section{4. 银行网格化管理的应用探析}

\section{1 资源共享基础设施的金融网格体系构建}

目前，各商业银行的业务数据系统基本实现了集中式处理，例如农业银行BoEing四期上 线后各项子系统更加协同。金融网格体系的构建，为实现软硬件资源共享、广泛开展存贷款 利率计算、业务报表统计、中间业务发展等均可提供良好的资源基础。这并不是废弃已有信 息资源, 也不是自下而上的分散式建设, 而是充分利用银行系统现有资源进一步优化性能, 提升应用、物理分散和逻辑集中, 从而改善应用环境, 实现信息资源互联互通, 显著提高业 务运行效率。网格中心可设置在总行或一级分行, 作为数据处理中心和信息汇集中心, 分别 连接各支行和网点的网格系统。此外, 该网格中心必须同时是一个金融认证中心, 为各支行 和网点分别设置身份认证, 为所涉统计、监管、审计等操作人员签发证书, 以验证请求者的 数字签名, 如此逐层次安全确认，才可以保障金融网格安全地互联互通和实现资源共享。

\section{2 应急人力资源网格调配}

应急人力资源一般是统称具有一定智力和体力劳动能力，能够及时发现、有效预防和妥 善处置突发事件。银行业中所需的应急人力资源突发事件主要是指人员紧缺以及精准营销分 流, 其中较为突出的是现金高柜中三人两柜制度, 另一方面则是职业预期不固定和高离职率 的存在性。网格调配基于五层沙漏结构, 使用层次模型的资源管理方式, 将各支行和网点具 体应急人力资源统一汇集成抽象服务形式，以解决具体问题。例如同一网点两个或以上柜员 同时缺岗, 需及时调配以保障该网点的日常运营。应急人力资源网格调配的具体运行是从应 急事件发生, 应急中心对此事件评定后做出调配任务后整合各应急部门方案并集合成统一的 调配方案, 并由应急中心统一指挥, 再将处置过后的事件案例输送至应急人力资源信息中心, 最终实现应急人力资源的高度共享和效用最大化。此外, 应急人力资源网格配置实现的是应 急时所需人力资源的共享, 能冲破跨网点、跨职能部门、甚至跨区域之间种种壁垒, 能即时 调配所需合适的人力资源或者服务, 实现应急人力资源的优化乃至无障碍调配。

应急人力资源网格强化履职管理, 汇聚分散式人力资源以实现资源共享, 所属网点、部 门甚至地理位置并不重要, 能够为突发事件提供大量资源组合, 以达到预期目标, 确保突发 事件中人力资源运行的安全性和稳定性, 并可有效提高解决危机的效率。 


\section{3 员工行为网格化管理}

银行业电子化程度不断提高, 但其正常运营仍主要取决于人的因素, 因此员工行为管理 一直是银行业管理的焦点所在。员工行为管理的核心是预防性管理, 重点不是解决问题而是 保持正确的员工行为，首先体现在正确完整的工作描述，分配或下达指令时则可视员工个人 情况提供实用有效的策略, 及时跟进时应使用有助于确认了解的语言, 而在处理事情时尤其 是面对员工异常行为表现出来的应是理解和尊重。

网格化管理作为一种新型管理模式，强调责任机制与协调机制，即每个成员都必须以服 务对象为导向, 负责其需求的全过程, 并打破各职能部门之间的限制, 通过资源的共享与协 调提高服务质量。针对员工行为的网格化管理, 细化明确员工行为管理职责、管理内容、管 理过程, 落实各层级人员责任, 构筑起全方位、全天候、全过程的责任共担的员工行为管理 体系。员工行为网格化管理可覆盖传统银行对个别差异员工行为的管理缺失, 应是能够按照 业务风险大小来分层经营管理，不仅增进服务流畅性，而且能够解决以往总分结构中信息不 对称引起的风险突出问题, 实现风控与效率兼顾。员工行为网格化管理不仅是系统管理和日 常业务的督导检查, 健全和严格落实完善各分支行、各部门、各岗位业务规章制度和操作流 程, 更是基于心理契约的人文关怀和有效激励机制以提升员工满意度, 并包含员工道德记录 和评价体系, 加强道德教育和业务培训, 引导员工自觉遵守规制。加强临柜员工导向的管理 可通过设立临柜员工服务内部专线等有效物理连接及时触发网格化机制实施。各商业银行可 推广团队建设式的网格化管理, 覆盖全员, 各员工对应着各自的格主和格友多元协作, 格内 定人明确角色和责任, 更有效地相互激励和共同成长。例如农行全面推广的 “三线一网格” 管理模式及应用系统，“三线” 即以纪检监督为保障、以案件风险防控为重点、以IT系统平 台为支撑, “一网格” 即以员工行为网格化管理为抓手, 落地生效以来行内员工行为管理工 作的主动性和实效性得到显著增强。

此外，网格化管理的本质在于服务，并非管控，因此网格必须界定在公共事物中，且必 须是受理型的通过触发机制和受理机制来发挥其作用, 从而引发网格管理系统的运作。因此, 网格化管理应显现出申请型, 而非主动的干涉。也因此, 员工行为网格化管理实施过程中, 需界定严格明确的边界, 不可侵入私人领域和侵犯个人隐私, 否则网格化管理会引发最大范 围内的排斥甚至反对，网格化管理也将失去其存在的合法性。

\section{5. 结语}

网格化管理是基于网格技术和网格化思想的新管理模式。本文从网格及网格化管理的内 涵和特征出发, 进而探讨网格化管理的结构和内容, 最后探讨网格化管理在银行业的应用, 选取资源共享基础设施的金融网格体系构建、应急人力资源调配、员工行为网格化管理等三 方面。本文进一步完善了网格化管理的理论基础, 并为银行业的网格化管理应用提供理论支 撑和借鉴建议，对银行的经营和发展具有重要的现实意义。

\section{References}

[1] Lv Weiping. The Practice and Exploration of Grid Management on Rural Commercial Banks -taking Ningbo Fenghua Rural Commercial Bank as an example[J]. Zhejiang Finance, vol.7, pp. 56-58, 2014.

[2] Yang Zonghui, Tian Ye. Rethink on Grid Management[J]. Jinan Journal(Philosophy \& Social Science Edition), vol.12, pp. 27-32, 2017.

[3] Zhang Shasha. A Research on the Grid Allocation Model of Human Resources for Emergency[D]. University of Electronic Science and Technology of China, 2013. 